\title{
Measurement of Inferior Vena Cava Collapsibility Index and Its Correlation to Central Venous Pressure in Adult Critically Ill Patients A Prospective Observational Study
}

\author{
Azza Ahmed Ameen Hafez* \\ Consultant of Anesthesia \& Intensive Care, Zagazig University Hospital, Egypt \\ *Corresponding author: Azza Ahmed Ameen Hafez, MD, Consultant of Anesthesia \& Intensive Care, Zagazig University Hospital, \\ Zagazig, Egypt
}

\begin{abstract}
Fluid therapy is an essential component part management of critically ill patients. Proper estimation of the amount of needed fluids is of great importance due to the well-established adverse effects of marked negative and positive fluids balance. Central venous pressure has been widely used by ICU physicians for volume status assessment. Several methods have been postulated for volume status assessment, among which is the inferior vena cava collapsibility index. As the inferior vena cava is a thin-walled capacitance vessel that adjusts to the body's volume status by changing its diameter depending on the total body fluid volume. Giving the fact that bedside ultrasonographic measurement of inferior vena cava diameters is an available, non-invasive, reproducible, and quiet easy-to-learn technique, it can provide a safe and quiet reliable replacement of central venous pressure measurement for assessment of volume status assessment.

The aim of this study: was to find statistical correlation between central venous pressure and caval index, as a step towards validating the above-mentioned replacement. 86 critically ill patients from ICU population were enrolled. Simultaneous measurements of central venous pressure and inferior vena cava collapsibility index were observed and recorded on four sessions. Patients were also grouped based on their mode of ventilation and central venous pressure values to compare the strength of correlation between various populations.

The results: showed that Inferior vena cava collapsibility index has significant inverse correlation with CVP value ( $r=-85, p$ value $<0.001$ at $95 \% \mathrm{CI}$ ) and it better correlated with mean arterial blood pressure and lactate clearance as compared to central venous pressure. However, it correlated better with CVP in spontaneously breathing patients $(r=-0.86, p$ value $<0.001)$ than in mechanically ventilated patients $(\mathrm{r}=-0.84$, $\mathrm{p}$ value $<0.001)$. Inferior vena cava collapsibility index has shown to correlate better with CVP value in lower values $\left(<10 \mathrm{cmH}_{2} \mathrm{O}\right)(\mathrm{r}=-0.8, \mathrm{p}$ value $<0.001)$ than in higher values $\left(\geq 10 \mathrm{cmH}_{2} \mathrm{O}\right)(\mathrm{r}=-0.6, \mathrm{p}$ value $<0.001)$. In

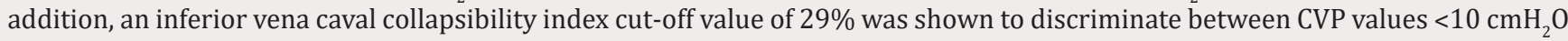
and values $\geq 10 \mathrm{cmH}_{2} \mathrm{O}$ with high Sensitivity (88.6\%) and specificity $(80.4 \%)$.
\end{abstract}

In conclusion: inferior vena cava collapsibility index has a strong inverse relationship with central venous pressure which is more pronounced at low central venous pressure values. Point-of-care ultrasono graphically measured inferior vena cava collapsibility index is very likely to be a good alternative to central venous pressure measurement with a high degree of precision and reproducibility. However, Wide scale studies are needed to validate it is use in different patient populations.

\section{Introduction}

The Surviving Sepsis Campaign consensus panel judged use of CVP targets to be recommended physiologic targets for resuscitation. Although there are limitations to CVP as a marker of intravascular volume status and response to fluids, a low CVP generally can be relied upon as supporting positive response to fluid loading [1].

Central venous pressure (CVP) is a key physiologic estimate of preload, which in turn helps define the intravascular fluid status. 
It is a particularly important parameter to measure in critically ill and injured patients who may require fluid resuscitation. Unfortunately, measurement of the CVP requires invasive central venous catheters that can be difficult or time-consuming to insert and are associated with complications. A non-invasive means of estimating the CVP would provide clinicians with an acceptable alternative [2]. Ultrasound is a tool that potentially could provide a rapid and non-invasive means of gauging preload and the need for fluid resuscitation. Because ultrasound machines are relatively light and portable, and many clinicians are trained in their use (e.g., emergency physicians, anesthesiologists, intensivists, and surgeons), the ability to non-invasively measure CVPs could extend patient monitoring capabilities to a variety of settings where direct measurements of the CVP are unavailable or impractical [2]. The inferior vena cava is a thin-walled compliant vessel that adjusts to the body's volume status by changing its diameter depending on the total body fluid volume. The vessel contracts and expands with each respiration. Negative pressure created by the inspiration of the patient increases venous return to the heart, briefly collapsing the IVC. Exhalation decreases venous return and the IVC returns to its baseline diameter. In states of low intravascular volume, the percentage collapse of the vessel will be proportionally higher than in intravascular volume overload states. This is quantified by the calculation of the caval index: IVC expiratory diameter - IVC inspiratory diameter, divided by IVC expiratory diameter $\times 100=$ caval index (\%) [3].

\section{Aim of the Work}

The aim of the work is to find relationship between inferior vena cava collapsibility index and central venous pressure in critically ill patients.

\section{Subjects and Methods}

It was prospective observational study All patients enrolled from patients admitted to surgical intensive care units at University hospitals.

Sample Size: The total number of patients admitted is 1800 patients per year. As the positive predictive value (PPV) $=70 \%$ at $80 \%$ power and $95 \%$ Confidence interval (CI) so the estimated sample size is 70 cases divided into two patient groups. (35 patients in each group) (EPI-INFO version 6)

\section{Subjects Included in the Study}

\section{a. Inclusion criteria:}

Critically ill patients admitted to trauma and surgical emergencies ICU and have the following criteria:

1- $\quad$ Age between 18 and 60 years

2- Have a properly positioned and functioning central venous catheter inserted into the internal jugular or subclavian vein for appropriate clinical indications.

\section{b. Exclusion criteria:}

1- Patients with cardiac disease (e.g. Heart failure, pulmonary hypertension, Corpulmonale)

2- $\quad$ Patients with liver disease (e.g. liver cirrhosis, liver cysts)

3- Patients with documented increased intra-abdominal pressure (above normal value: 7-10 $\mathrm{cmH}_{2} \mathrm{O}$ )

4- Patients with increased intrathoracic pressure (e.g. Pneumothorax, pleural effusion)

5- Patients requiring circulatory support (vasopressors or inotropic support).

6- Patients in whom the required ultrasound examination of the inferior vena cava would not be possible or appropriate (e.g. Burst abdomen, extensive burns, excess bowel gases).

All patients were subjected to thorough clinical evaluation with special emphasis on:

1- Full history.

2- examination of the patient

3- Measurement of intra-abdominal pressure. (using the standard method: indirect measurement via a catheter connected to the urinary bladder. The bladder was drained using a urinary catheter before measuring intra-abdominal pressure, and 50-100 mL normal saline was injected into the bladder; the distal portion was clamped. A 16-gauge needle was inserted into the output of the urinary catheter the needle was connected to a three-way tap and water manometer. Symphysis pubis was taken as a reference point. After being filled with saline, the patient side of the manometer was opened. The intra-abdominal pressure results were measured with each patient reading and recorded in $\mathrm{cmH}_{2} \mathrm{O}$. Intra-abdominal hypertension (IAH) is defined as a sustained intra-abdominal pressure $\geq 12 \mathrm{mmHg}\left(\geq 16.3 \mathrm{cmH}_{2} \mathrm{O}\right.$ ).

4- Laboratory investigations: complete blood picture (CBC), liver function tests (LFT), kidney function tests (KFT) and Bleeding profile, arterial blood gases (ABG) and urine output.

\section{5- Radiological study: plain X ray chest radiograph}

\section{Steps of Performance and Technique Used}

a. After fulfillment of the above criteria and prerequisites, each eligible patient had simultaneous measurement of central venous pressure and Inferior vena cava collapsibility index on four different sessions; the first was on admission, the second was at the end of the first six hours after admission, the third was at 12 hours after admission and the fourth was at 24 hours 
after admission.

b. Patients were divided into two groups based on their central venous pressure values: the first group (CVP > 10 $\mathrm{cmH}_{2} \mathrm{O}$ ) and second group (CVP $\geq 10 \mathrm{cmH}_{2} \mathrm{O}$ )

c. Measurements will be done as follows:

d. Central venous pressure measurement

\section{Steps for Central Venous Pressure Measurement Using Water Manometer}

a. Ensure all intravenous infusions running though the manometer line are stopped

b. Zero the manometer

c. Close the three-way tap to the patient, opening the tap between the fluid and manometer

d. Allow the manometer to fill with fluid to a level beyond the expected pressure

e. Do not fill the line so far that the air filter becomes wet

f. Close the tap to the fluid source, opening the manometer to the patient

g. Watch the fluid level change. It should fall until gravity pressure equals the pressure from the central veins. When the fluid stops falling, read the CVP measurement Inferior vena cava collapsibility index calculation.

A. Was done by researchers of this study and attending ICU physicians after receiving adequate training in the form of a focused course on ultrasonographic imaging of the inferior vena cava. While the patient is in supine position, using Siemens Vacuson ${ }^{\circledR}$ X300 premium edition sector transducer (1.4-5 MHz) (Siemens Medical Solutions USA Inc. CA, USA). The probe is placed $2 \mathrm{~cm}$ caudal to the diaphragm in the subxiphoid area (subxiphoid view) to visualize the inferior vena cava and measuring its maximal diameters during end-inspiration and end-expiration just proximal to the hepatic veins using M-Mode scan.

B. Diameters of the inferior vena cava will be measured during one respiratory cycle and recorded as follows:

a. Maximum IVC diameter $\left(\mathrm{IVC}_{\max }\right)$ : maximum diameter measured during distension of the IVC

b. Minimum IVC diameter $\left(\mathrm{IVC}_{\min }\right)$ : minimum diameter measured during collapse of the IVC

c. Inferior vena cava collapsibility index (CI) will be calculated as follows:

Collapsibility index $(\mathrm{CI})=\left(\right.$ Maximum IVC diameter $\left(\mathrm{IVC}_{\max }\right)$ Minimum IVC diameter $\left(\mathrm{IVC}_{\text {min }}\right)$ )/ (Maximum IVC diameter $\left(\mathrm{IVC}_{\text {max }}\right)$ )

\section{Statistically Analyzed}

Collected data will be plotted and statistically analyzed using appropriate statistical software to define statistical significance of correlation (Figure 1).
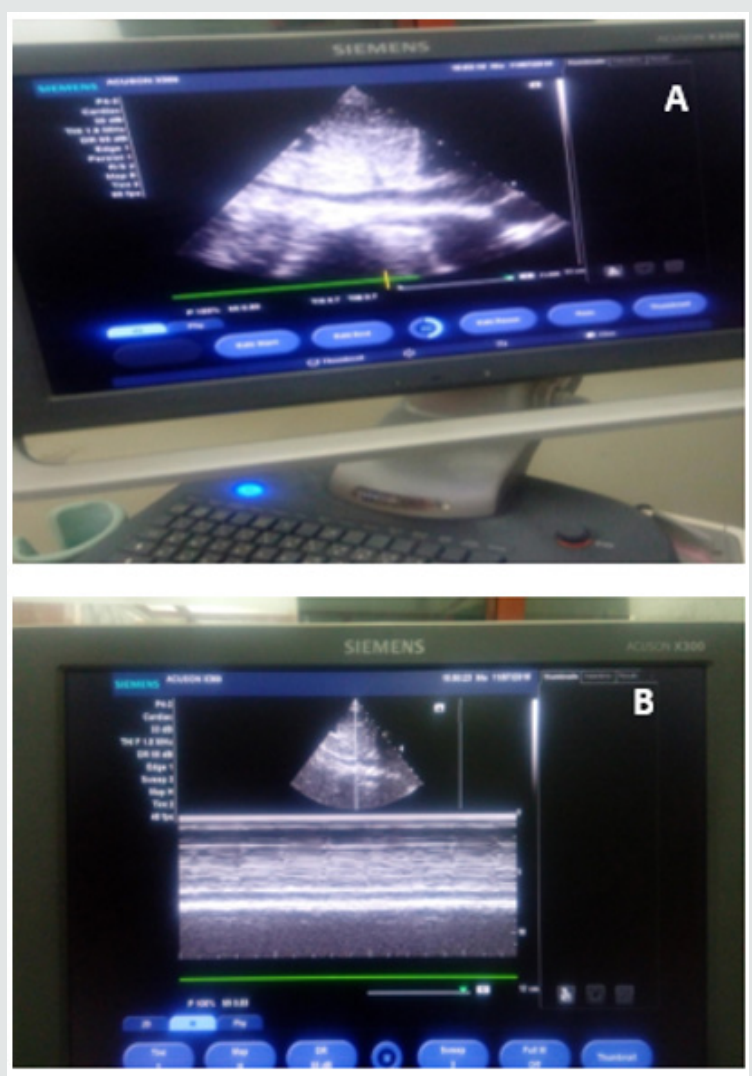

Figure 1: Ultaronographic imaging of the IVC in the subxiphoid view using Siemens Vacuson ${ }^{\circledR}$ X300 premium edition sector transducer (Siemens Medical Solutions USA Inc. CA, USA) in intensive care unit). (A) 2D-mode imaging of the IVC at its junction with the right atrium. Note the joining of the hepatic vein with the IVC. (B) Real time M-Mode scanning of the IVC to measure its inspiratory and expiratory diameters just proximal to the hepatic vein.

\section{Results}

A total of 86 patients who were admitted to trauma and surgical emergency intensive care unit at university hospitals were eligible for enrollment in this study over the period of two years (October 2017- October 2019). Of these patients 62 were males (72.1\%). Mean age was 36.8 years. the main cause of ICU admission was polytrauma $(n=49,57 \%)$. On ICU admission, mean CVP was 7.5 $\mathrm{cmH}_{2} \mathrm{O}$. Mean systolic blood pressure was $115.14 \mathrm{mmHg}$. Mean serum lactate level was $2.29 \mathrm{mmol} / \mathrm{L}$. According to mode of ventilation in the baseline reading patients were divided into two groups. The first group is spontaneously breathing patients (10 patients, $11.6 \%$ of patient sample). Mean CVP value is $4.25 \pm 4.5 \mathrm{cmH}_{2} \mathrm{O}$ while mean inferior vena cava collapsibility index is $0.46 \pm 0.22 \%$. The second 
group is mechanically ventilated patients (76 patients, $89.4 \%$ ). Mean CVP value is $8.81 \pm 4.642 \mathrm{cmH}_{2} \mathrm{O}$ while mean inferior vena cava collapsibility index is $0.32 \pm 0.184 \%$. According to CVP value, Patient readings were divided into 2 groups.

The first group included all patients reading with CVP value less than $10 \mathrm{cmH}_{2} \mathrm{O}$, while the second group included all patient readings with CVP value equal to or more than $10 \mathrm{cmH}_{2} \mathrm{O}$. Correlation coefficients in each group Inferior vena cava collapsibility index has shown to correlate better with CVP value in lower values $\left(<10 \mathrm{cmH}_{2} \mathrm{O}\right)(\mathrm{r}=-0.8, \mathrm{p}$ value $<0.001)$ than in higher values $(\geq 10$ $\left.\mathrm{cmH}_{2} \mathrm{O}\right)(\mathrm{r}=-0.6, \mathrm{p}$ value $<0.001)$. A linear regression analysis model was carried out to ascertain to which extent inferior vena cava collapsibility index can predict CVP value. A strong correlation was found between inferior vena cava collapsibility index and CVP value $(r=0.85)$ and the regression model predicted $72 \%$ of the variance. Receiver Operator Characteristics (ROC) curve was plotted for evaluation of sensitivity and specificity of inferior vena cava collapsibility index in estimating CVP value. Inferior vena caval collapsibility index cut-off value of $29 \%$ discriminates between CVP values $<10 \mathrm{cmH}_{2} \mathrm{O}$ [collapsibility index $>29 \%$ ] and values $\geq 10$ $\mathrm{cmH}_{2} \mathrm{O}$ [collapsibility index $<29 \%$ ] with high Sensitivity (88.6\%) and specificity $(80.4 \%)$ (Tables $1-9)$.

Table 1: Patient data of study population.

\begin{tabular}{|c|c|c|c|c|}
\hline \multirow{2}{*}{ Age } & Minimum & Maximum & Mean & Std. Deviation \\
\hline & 22 & 58 & 36.84 & 9.64 \\
\hline \multirow{4}{*}{ Gender } & & \multicolumn{2}{|c|}{ Frequency } & $\%$ \\
\hline & Female & \multicolumn{2}{|c|}{24} & 27.9 \\
\hline & Male & \multicolumn{2}{|c|}{62} & 72.1 \\
\hline & Total & \multicolumn{2}{|c|}{86} & 100 \\
\hline \multirow{4}{*}{ Mode of ventilation } & \multirow{4}{*}{$\begin{array}{c}\text { Mechanical ventilation } \\
\text { Spontaneous breathing } \\
\text { Total }\end{array}$} & \multicolumn{2}{|c|}{ Frequency } & $\%$ \\
\hline & & \multicolumn{2}{|c|}{76} & 88.4 \\
\hline & & \multicolumn{2}{|c|}{10} & 11.6 \\
\hline & & \multicolumn{2}{|c|}{86} & 100 \\
\hline
\end{tabular}

Table 2: Correlation coefficients for CVP value and inferior vena cava collapsibility index with other measured hemodynamic parameters in study population. ${ }^{* *}$. Correlation is significant at the 0.01 level (2-tailed). *. Correlation is significant at the 0.05 level (2-tailed).

\begin{tabular}{|c|c|c|c|c|c|c|c|c|c|}
\hline Parameter & SYS BP & DIA BP & $\begin{array}{c}\text { MEAN } \\
\text { BP }\end{array}$ & CVP & IVC MAX & IVC MIN & IVC CI & $\begin{array}{c}\text { LACTATE CLEAR- } \\
\text { ANCE }\end{array}$ & UOP \\
\hline \multirow{2}{*}{ CVP, Pearson Correlation Sig. } & $.353^{* *}$ & $.391^{* *}$ & $.401^{* *}$ & 1 & $.623^{* *}$ & $.877^{* *}$ & $-.852^{* *}$ & $.243^{* *}$ & $-.265^{* *}$ \\
(2-tailed) N & 0 & 0 & 0 & & 0 & 0 & 0 & 0 & 0 \\
IVC Ci Pearson Correlation Sig. & 344 & 344 & 344 & 344 & 344 & 344 & 344 & 258 & 258 \\
(2-tailed) N & $-.348^{* *}$ & $-.407^{* *}$ & $-.411^{* *}$ & $-.852^{* *}$ & $-.538^{* *}$ & $-.923^{* *}$ & 1 & $-.320^{* *}$ & $.234^{* *}$ \\
& 0 & 0 & 0 & 0 & 0 & 0 & & 0 & 0 \\
\end{tabular}

Abbreviation set for table (4): SYS BP: Systolic Blood Pressure. DIA BP: Diastolic Blood Pressure. MEAN BP: Mean Blood Pressure. CVP: Central Venous Pressure. IVC MAX: Maximal Diameter of Inferior Vena Cava. IVC MIN: Minimal Diameter of Inferior Vena Cava. IVC Ci: Inferior Vena Cava Collapsibility index. LACTATE CLEARANCE: percentage of lactate clearance since previous reading. UOP: Urine Output. Balance: net fluid Balance.

Table 3: Descriptive statistical data of the spontaneously breathing patient group.

\begin{tabular}{|c|c|c|c|}
\hline Parameter (Unit) & N & Mean & Std. Deviation \\
\hline Age (years) & 10 & 37.7 & 11.1 \\
\hline IAP $\left(\mathrm{cmH}_{2} \mathrm{O}\right)$ & 40 & 8.5 & 2.39 \\
\hline HR (beat/minute) & 40 & 104.45 & 14.4 \\
\hline SYS BP (mmHg) & 40 & 111.33 & 16.32 \\
\hline DIA BP (mmHg) & 40 & 67.03 & 17.74 \\
\hline
\end{tabular}




\begin{tabular}{|c|c|c|c|}
\hline MEAN BP (mmHg) & 40 & 81.75 & 16.61 \\
\hline CVP $\left(\mathrm{cm} \mathrm{H}_{2} \mathrm{O}\right)$ & 40 & 4.13 & 4.51 \\
\hline IVC MAX (mm) & 40 & 16.56 & 5.56 \\
\hline IVC MIN (mm) & 40 & 9.48 & 0.22 \\
\hline IVC Ci (percentage) & 40 & 0.46 & 1.67 \\
\hline LACTATE (mmol/L) & 40 & 3.63 & 409.09 \\
\hline CLEARANCE (\%) & 30 & 6006.78 & 608.17 \\
\hline FLUID (mL) & 30 & 522.63 & 505.07 \\
\hline UOP (mL) & 30 & 356.28 & 599.14 \\
\hline BALANCE (mL) & 30 & \\
\hline
\end{tabular}

Abbreviation set for table (11) IAP: Intra-Abdominal Pressure. HR: Heart Rate. SYS BP: Systolic Blood Pressure. DIA BP: Diastolic Blood Pressure. MEAN BP: Mean Blood Pressure. CVP: Central Venous Pressure. IVC MAX: Maximal Diameter of Inferior Vena Cava. IVC MIN: Minimal Diameter of Inferior Vena Cava. IVC Ci: Inferior Vena Cava Collapsibility index. LACTATE: serum Lactate level. CLEARANCE: percentage of lactate clearance since previous reading. Fluid: amount of infused intravenous Fluids. UOP: Urine Output. Balance: net fluid Balance.

Table 4: Descriptive statistical data of the mechanically ventilated patient group.

\begin{tabular}{|c|c|c|c|}
\hline Parameter (Unit) & N & Mean & Std. Deviation \\
\hline Age (years) & 76 & 36.72 & 9.5 \\
\hline IAP (cmH ${ }_{2}$ ) & 304 & 9.07 & 16.68 \\
\hline HR (beat/minute) & 304 & 97.6 & 14.4 \\
\hline SYS BP (mmHg) & 304 & 115.56 & 13.15 \\
\hline DIA BP (mmHg) & 304 & 72.03 & 12.74 \\
\hline MEAN BP (mmHg) & 304 & 86.55 & 4.64 \\
\hline CVP (cmH ${ }_{2}$ ) & 304 & 8.18 & 3.01 \\
\hline IVC MAX (mm) & 304 & 20.09 & 4.95 \\
\hline IVC MIN (mm) & 304 & 13.91 & 0.18 \\
\hline IVC Ci (percentage) & 304 & 0.32 & 1.18 \\
\hline Lactate (mmol/L) & 304 & 2.33 & 331.01 \\
\hline Fluid (mL) & 228 & 860.26 & 322.88 \\
\hline UOP (mL) & 228 & 699.56 & 405.6 \\
\hline Balance (mL) & 228 & 160.7 & 37 \\
\hline Clearance (\%) & 228 & -11.04 & \\
\hline
\end{tabular}

Abbreviation set for table (12) IAP: Intra-Abdominal Pressure. HR: Heart Rate. SYS BP: Systolic Blood Pressure. DIA BP: Diastolic Blood Pressure. MEAN BP: Mean Blood Pressure. CVP: Central Venous Pressure. IVC MAX: Maximal Diameter of Inferior Vena Cava. IVC MIN: Minimal Diameter of Inferior Vena Cava. IVC Ci: Inferior Vena Cava Collapsibility index. LACTATE: serum Lactate level. CLEARANCE: percentage of lactate clearance since previous reading. Fluid: amount of infused intravenous Fluids. UOP: Urine Output. Balance: net fluid Balance.

Table 5: Correlation coefficients in spontaneously breathing patient group. ${ }^{* *}$. Correlation is significant at the 0.01 level (2-tailed). * Correlation is significant at the 0.05 level (2-tailed).

\begin{tabular}{ccc|c|c} 
Parameter & Cvp & Lactate & Clearance & Balance \\
IVC CiPearson CorrelationSig. (2-tailed) & $-.866^{* *}$ & $.629^{* *}$ & -0.069 & -0.5 \\
& 0 & 0 & 0.77 & 0.545
\end{tabular}

Abbreviation set for table (8). CVP: Central Venous Pressure. IVC Ci: Inferior Vena Cava Collapsibility index. LACTATE: serum Lactate level. CLEARANCE: percentage of lactate clearance since previous reading. Balance: net fluid Balance. 
Table 6: Correlation coefficients in mechanically ventilated patient group. **. Correlation is significant at the 0.01 level (2-tailed). * Correlation is significant at the 0.05 level (2-tailed).

\begin{tabular}{|c|c|c|c|c|}
\hline Parameter & CVP & Lactate & Clearance2 & Balance \\
\hline \multirow{2}{*}{ IVC CiPearson CorrelationSig. (2-tailed) } & $-.842^{* *}$ & $.243^{* *}$ & $-.23^{* *}$ & $-.338^{* *}$ \\
\hline & 0 & 0 & 0 & 0 \\
\hline
\end{tabular}

Abbreviation set for table (9). CVP: Central Venous Pressure. IVC Ci: Inferior Vena Cava Collapsibility index. LACTATE: serum Lactate level. CLEARANCE: percentage of lactate clearance since previous reading. Balance: net fluid Balance.

Table 7: Correlation coefficients in lower CVP values $\left(<10 \mathrm{cmH}_{2} \mathrm{O}\right)$ group. **. Correlation is significant at the 0.01 level (2-tailed). * Correlation is significant at the 0.05 level (2-tailed).

\begin{tabular}{|c|c|c|c|c|c|}
\hline Parameter & Mean BP & CVP & LACTATE & Clearance & Balance \\
\hline IVC Ci, Pearson Correlation Sig. (2-tailed) & $\begin{array}{c}-0.376^{* *} \\
0\end{array}$ & $\begin{array}{c}-0.80^{* *} \\
0\end{array}$ & $\begin{array}{c}0.534^{* *} \\
0\end{array}$ & $\begin{array}{c}-0.353^{* *} \\
0\end{array}$ & $\begin{array}{c}-0.94^{*} \\
0.04\end{array}$ \\
\hline
\end{tabular}

Abbreviation set for table (10). MEAN BP: Mean Blood Pressure CVP: Central Venous Pressure. IVC Ci: Inferior Vena Cava Collapsibility index. LACTATE: serum Lactate level. CLEARANCE: percentage of lactate clearance since previous reading. Balance: net fluid Balance.

Table 8: Correlation coefficients in higher CVP values $\left(\geq 10 \mathrm{cmH}_{2} \mathrm{O}\right)$ group. **. Correlation is significant at the 0.01 level (2-tailed). * Correlation is significant at the 0.05 level (2-tailed).

\begin{tabular}{|c|c|c|c|c|c|}
\hline Parameter & Mean BP & CVP & Lactate & Clearance & Balance \\
\hline \multirow{2}{*}{ IVC Ci Pearson Correlation Sig. (2-tailed) } & -0.62 & $-0.603^{* *}$ & $-0.3^{* *}$ & 0.07 & -0.97 \\
\cline { 2 - 7 } & 0.062 & 0 & 0 & 0.866 & 0.05 \\
\hline
\end{tabular}

Abbreviation set for table (11). MEAN BP: Mean Blood Pressure CVP: Central Venous Pressure. IVC Ci: Inferior Vena Cava Collapsibility index. LACTATE: serum Lactate level. CLEARANCE: percentage of lactate clearance since previous reading. Balance: net fluid Balance.

Table 9: Regression analysis model for correlation was found between inferior vena cava collapsibility index and CVP value (a\&b).

\begin{tabular}{|c|c|c|c|c|}
\hline Model & R & R Square & Adjusted R Square & Std. Error of the Estimate \\
\hline & $0.852 \mathrm{a}$ & 0.727 & 0.726 & 2.5425 \\
\hline
\end{tabular}

Predictors: (Constant), IVC Ci

\begin{tabular}{|c|c|c|c|c|c|}
\hline \multicolumn{2}{|c|}{ Model } & \multicolumn{2}{|c|}{ Unstandardized Coefficients } & Standardized Coefficients & t \\
\hline & B & Std. Error & & \\
(Constant) IVC Ci & 4.833 & 0.272 & -0.852 & 54.44 & 0 \\
& -2.087 & 0.7 & -30.4 & 0 \\
\hline
\end{tabular}

Dependent Variable: CVP

\section{Receiver Operator Characteristics (ROC) Curve}

Receiver Operator Characteristics (ROC) curve was plotted for evaluation of sensitivity and specificity of inferior vena cava collapsibility index in estimating CVP value (Figure 2 \&Table 10).

Table 10: Sensitivity and Specificity of inferior vena cava collapsibility index in estimating CVP value.

(a)

\begin{tabular}{|c|c|c|c|c|}
\hline \multicolumn{5}{|c|}{ Area Under the Curve } \\
\hline \multicolumn{5}{|c|}{ Test Result Variable(s): IVC_Ci } \\
\hline \multirow{2}{*}{ Area } & \multirow{2}{*}{ Cutoff } & \multirow{2}{*}{$P$} & 95\% Confidence Interval & \multirow{2}{*}{ Upper Bound } \\
\hline & & & Lower Bound & \\
\hline 0.954 & $<0.29$ & $0.00^{* *}$ & 0.96 & 0.993 \\
\hline
\end{tabular}

Citation: Azza Ahmed Ameen Hafez. Measurement of Inferior Vena Cava Collapsibility Index and Its Correlation to Central Venous Pressure in Adult Critically Ill Patients A Prospective Observational Study. Glob J Anes \& Pain Med 3(4)-2020. GJAPM.MS.ID.000170. DOI: 10.32474/ 
(b)

\begin{tabular}{|c|c|c|c|c|c|c|c|c|}
\hline \multirow[b]{4}{*}{ IVCci } & \multirow[b]{3}{*}{$>0.29$} & \multirow[b]{3}{*}{$\mathrm{N}$} & \multicolumn{2}{|c|}{ CVP_GROUP } & \multirow{2}{*}{ Total } & \multirow{2}{*}{$\mathrm{X} 2$} & \multirow{2}{*}{$\mathbf{P}$} & \multirow{2}{*}{ Kappa agreement } \\
\hline & & & $<10 \mathrm{cmH}_{2} \mathrm{o}$ & $\geq 10 \mathrm{cmH}_{2} \mathrm{o}$ & & & & \\
\hline & & & 41 & 4 & 45 & \multirow{4}{*}{39.57} & \multirow{4}{*}{$0.00^{* *}$} & \multirow{4}{*}{0.67} \\
\hline & \multirow{3}{*}{$<0.29$} & $\%$ & $80.40 \%$ & $11.40 \%$ & $52.30 \%$ & & & \\
\hline & & $\mathrm{N}$ & 10 & 31 & 41 & & & \\
\hline & & $\%$ & $19.60 \%$ & $88.60 \%$ & $47.70 \%$ & & & \\
\hline \multirow{2}{*}{ Total } & & $\mathrm{N}$ & 51 & 35 & 86 & & & \\
\hline & & $\%$ & $100.00 \%$ & $100.00 \%$ & $100.00 \%$ & & & \\
\hline
\end{tabular}

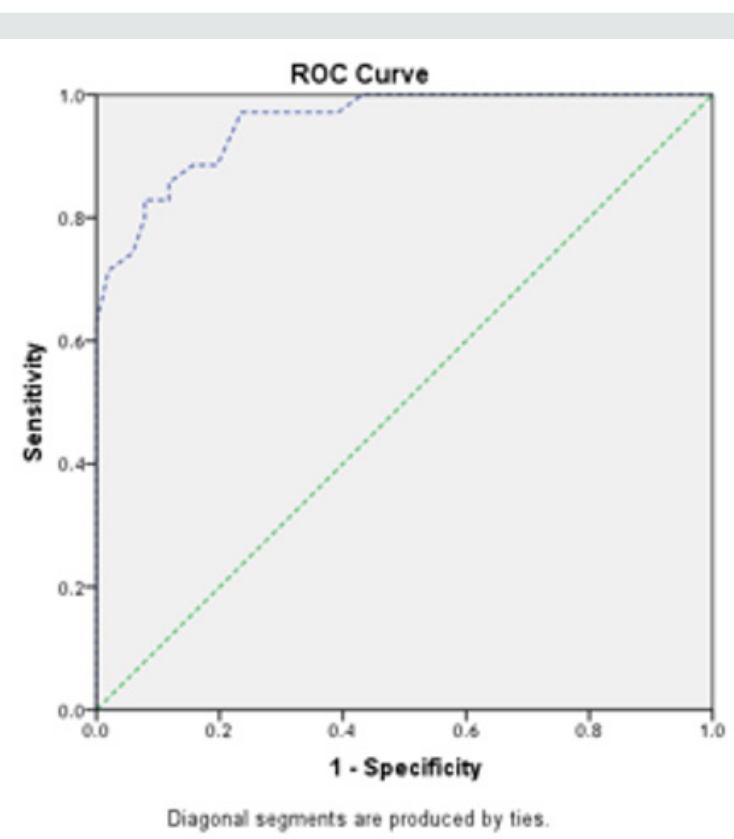

Figure 2: Receiver Operator Characteristics (ROC) Curve.

\section{Discussion}

In a recent meta-analysis by Eskesen et al, lower CVP values (<8 $\mathrm{mmHg}$ ) predicted fluid responsiveness. Meanwhile intermediate (8-12 $\mathrm{mmHg}$ ) and higher (>12 $\mathrm{mmHg}$ ) ranges of CVP values did not predict fluid responsiveness. However, predictive values were generally low for all specific CVP values [4]. Barbier and colleagues conducted a study on Twenty-three mechanically ventilated patients with acute circulatory failure related to sepsis to evaluate the extent to which respiratory changes in inferior vena cava (IVC) diameter can be used to predict fluid responsiveness. They specified a threshold inferior vena cava collapsibility index of $18 \%$ to discriminate between responders and non-responders with $90 \%$ sensitivity and $90 \%$ specificity. A strong relation ( $r=0.9$ ) was observed between inferior vena cava collapsibility index at baseline and the increase in cardiac index following blood volume expansion, while baseline central venous pressure did not accurately predict fluid responsiveness. They concluded that respiratory change in IVC diameter is an accurate predictor of fluid responsiveness in septic patients [5]. A meta-analysis by Zhang et al. [6] demonstrated that respiratory variation of inferior vena cava diameter measured with point-of-care ultrasonography is of great value in predicting fluid responsiveness, particularly in patients on controlled mechanical ventilation and in patients resuscitated with colloids [6].

Muller et al. [7] conducted a study over forty patients with acute circulatory failure and spontaneous breathing to determine ability of inferior vena cava collapsibility index to predict fluid responsiveness. Response to fluid challenge was defined as a $15 \%$ increase of subaortic velocity time index (VTI) measured by transthoracic echocardiography. They concluded that in spontaneously breathing patients with acute circulatory failure, high inferior vena cava collapsibility index values (> 40\%) are usually associated with fluid responsiveness while low values $(<40 \%)$ do not exclude fluid responsiveness [7]. Our study showed that Inferior vena cava collapsibility index has significant inverse correlation with CVP value $(r=-0.85$, $\mathrm{p}$ value $<0.001$ at $95 \% \mathrm{CI}$ ) and it better correlated with mean arterial blood pressure and lactate clearance as compared to central venous pressure. However, it correlated slightly better with CVP in spontaneously breathing patients $(r=-0.86$, $p$ value $<0.001)$ than in mechanically ventilated patients $(r=-0.84$, $p$ value $<0.001)$. Inferior vena cava collapsibility index has shown to correlate better with CVP value in lower values $\left(<10 \mathrm{cmH}_{2} \mathrm{O}\right)(\mathrm{r}=-0.8, \mathrm{p}$ value $<0.001)$ than in higher values $(\geq 10$ $\mathrm{cmH}_{2} \mathrm{O}$ ) ( $\mathrm{r}=-0.6, \mathrm{p}$ value $\left.<0.001\right)$. We also concluded an inferior vena caval collapsibility index cut-off value of $29 \%$ to discriminate between CVP values $<10 \mathrm{cmH}_{2} \mathrm{O}$ and values $\geq 10 \mathrm{cmH}_{2} \mathrm{O}$ with high Sensitivity (88.6\%) and specificity (80.4\%).

Worapratya et al. conducted a study on 30 adult patients who presented to the emergency room between September 2012 and November 2012. Patients with postcardiac arrest, intraabdominal compartment syndrome, ventilated patients, pregnant patients, and any patient who was not suitable for bedside ultrasonographic measurement of the IVC diameters were excluded in their study. Observed correlations of the CVP measurement with the ultrasound IVC caval index were $\mathrm{r}=-0.721(\mathrm{P}=0.000)$ by $2 \mathrm{D}$-mode ultrasound and $\mathrm{r}=-0.647(\mathrm{P}=0.001)$ by M-mode. The sensitivity and specificity of the caval index to predict the CVP were calculated. Results showed 
that the cut-off points of the caval index were 30\% for CVP levels of $<10 \mathrm{cmH}_{2} \mathrm{O}$ (Sensitivity $85.7 \%$ and Specificity $95.7 \%$ ), 20\% for 10 $15 \mathrm{cmH}_{2} \mathrm{O}$ (Sensitivity $33.3 \%$ and Specificity 62.5\%), and $10 \%$ for CVP levels $>15 \mathrm{~cm} \mathrm{H}_{2} \mathrm{O}$ (Sensitivity $76.5 \%$ and Specificity $76.9 \%$ ) [8]. These results are comparable to the results of the current study. Wiryana et al. [9] conducted a study over 70 patients from September to October of 2016 at the intensive care unit (ICU) of Sanglah General Hospital at Bali Island, Indonesia. Patients whose age ranged from 18 to 64 years, were able to lie supine, with normal body mass index and were mechanically ventilated were included. Patients who refused to join the study, on vasoconstrictor or vasodilator drugs, with cardiac dysfunction, increasing abdominal pressure and intrathoracic pressure were excluded. They found a very strong negative correlation between CVP and inferior vena cava collapsibility index $(r=-0.854 ; p<0.001)$ [9]. This agrees with the results of our study. A study conducted by Ilyas et al over 100 adult medical intensive care unit (ICU) patients over a period of three months. 47 patients were on mechanical ventilation. Patients with clinical signs of elevated abdominal pressure, moderate to severe tricuspid regurgitation, CVP inserted for more than 24 hours, and patients in whom the supine position was contraindicated were excluded. They found a strong inverse linear correlation between the central venous pressure and the inferior vena cava collapsibility index, which was statistically significant $(r=-0.827$, $p<0.0005$ ) [10]. These results are concordant with the results of the current study. Karacabey et al. conducted a study Between June and October 2012 over 83 patients who were treated in the critical care area. All of the patients were mechanically ventilated and had a central venous line. They excluded Patients who were younger than 18 years old, not intubated, trauma patients, pregnant patients, and/or having known or newly diagnosed intraabdominal hypertension (defined as an intraabdominal pressure $\geq 12 \mathrm{mmHg}$ ). IVC collapsibility measurements showed an inverse correlation with CVP measurements $(\mathrm{p}<0.01 ; \mathrm{r}=0.68)$ [11]. The results are comparable to the results posted in the current study.

In another study by Stawicki and colleagues, 124 patients were enrolled. $56(45.2 \%)$ of them were on mechanical ventilation. Eligible patients were subjected to Intensivist-performed bedside ultrasonography (INBU) examinations to evaluate hemodynamic status of SICU patients, focusing on correlations between IVC-CI and CVP. IVC-CI measurements were grouped by range $(<0.20$, 0.20 to 0.60 , and $>0.60$ ) and analyzed for presence of substantial differences in CVP between the three IVC-CI groupings. These IVCCI cut-off values were determined arbitrarily, with the intention of selecting groups with high, intermediate, and low collapsibility indices. CVP values were also grouped into three ranges: $<7 \mathrm{mmHg}$, 7 to $12 \mathrm{mmHg}$, and $>12 \mathrm{mmHg}$. These values were chosen based on ranges set by fluid management policy in their surgical intensive care unit (SICU). They concluded that There was a significant, although weak correlation between IVC-CI and CVP measurements for continuous data $(r=0.315 ; \mathrm{p}=0.007)$ and that IVC-CI appears to correlate best with CVP in the setting of low $(<0.20)$ and high $(>0.60)$ collapsibility ranges. The authors suggested that measurements of IVC-CI by INBU can provide a useful guide to noninvasive volume status assessment in SICU patients. However the authors stated there are several factors that could have possibly weakened this correlation such as the high prevalence of increased intraabdominal pressures in patient population and the possibility of presence of elevated pulmonary artery pressures, tricuspid or pulmonic valve disease, and right and left ventricular dysfunction in an unknown number of patients [12]. All of these factors were excluded in the current study as previously mentioned in our methodology. In summary, inferior vena cava collapsibility index is found to have a remarkable correlation with central venous pressure value and may provide a useful tool for rapid and reliable estimation of volume status in patients particularly in the quiet busy environment of intensive care units and emergency departments where often quick decisions about fluid management are required.

\section{Conclusion}

Inferior vena cava collapsibility index has a strong inverse relationship with central venous pressure which is more pronounced in the setting of low central venous pressure values. A caval index of $29 \%$ provides a cut-off value which may reliably discriminate between lower $\left(<10 \mathrm{cmH}_{2} \mathrm{O}\right)$ and higher $\left(\geq 10 \mathrm{cmH}_{2} \mathrm{O}\right)$ CVP values. Owing to the fact that ultrasonographic measurement of caval index is a simple and noninvasive safe technique which avoids many complications and contraindications of central venous access, and that the caval index appears to be more correlated with fluid responsiveness, point-of-care ultrasonographically-measured inferior vena cava collapsibility index is very likely to be a good alternative to central venous pressure measurement with a high degree of precision and reproducibility. However, wide scale studies are needed to validate its use in different patient populations.

\section{References}

1. Dellinger RP, Levy MM, Rhodes A, Annane D, Gerlach H, et al. (2013) Surviving Sepsis Campaign: International Guidelines for Management of Severe Sepsis and Septic Shock: 2012. Critical care medicine 41 (2): 580-637.

2. De Lorenzo RA, Morris MJ, Williams JB, Haley TF, Straight TM et al. (2012) Does a simple bedside sonographic measurement of the inferior vena cava correlate to central venous pressure? The Journal of Emergency Medicine 42(4): 429-436.

3. Goldflam K, Saul T, Lewiss R (2011) Focus On: Inferior Vena Cava Ultrasound. American College of Emergency Physicians.

4. Eskesen TG, Wetterslev M, Perner A (2015) Systematic review including re-analyses of 1148 individual data sets of central venous pressure as a predictor of fluid responsiveness. Intensive Care Med 42: 324-332

5. Barbier C, Loubires Y, Schmit C, Hayon J, Ricôme JL, et al. (2004) Respiratory changes in inferior vena cava diameter are helpful in predicting fluid responsiveness in ventilated septic patients. Intensive Care Med 30: 1740-1746. 
6. Zhang Z, Xu X, Ye S, Xu L (2014) Ultrasonographic measurement of the respiratory variation in the inferior vena cava diameter is predictive of fluid responsiveness in critically ill patients: systematic review and meta-analysis. ultrasound in medicine and biology 40(5): 845-853.

7. Muller L, Bobbia X, Toumi M, Louart G, Molinari N et al. (2012) Respiratory variations of inferior vena cava diameter to predict fluid responsiveness in spontaneously breathing patients with acute circulatory failure: need for a cautious use. Critical Care 16: 188.

8. Worapratya P, Anupat A, Suwannanon R, Wuthisuthimethawee P (2014) Correlation of caval index, inferior vena cava diameter, and central venous pressure in shock patients in the emergency room. Open Access Emergency Medicine 6: 57-62.

9. Wiryana M, Sinardja K, Wayan A, Senapathi TG, Widnyana MG, et al. (2017) Central venous pressure correlates with inferior vena cava collapsibility index in patients treated in intensive care unit. Bali Journal of Anesthesiology 1(1): 7-9.

10. Ilyas A, Ishtiaq W, Assad S, Ghazanfar H, Mansoor S, et al. (2017) Correlation of IVC diameter and collapsibility index with central venous pressure in the assessment of intravascular volume in critically ill patients. Cureus 9(2): 1025.

11. Karacabey S, Sanri E, Guneysel O (2016) A Non-invasive method for assessment of intravascular fluid status: Inferior vena cava diameters and collapsibility index. Pak J Med Sci 32(4): 836-840.

12. Stawicki SP, Adkins EJ, Eiferman DS, Evans DC, Ali NA, et al. (2013) Prospective evaluation of intravascular volume status in critically ill patients: Does inferior vena cava collapsibility correlate with central venous pressure? J Trauma Acute Care Surg 76(4): 956-964.

\section{CC) (P) This work is licensed under Creative}

To Submit Your Article Click Here:

Submit Article

DOI: $10.32474 /$ GJAPM.2020.03.000170

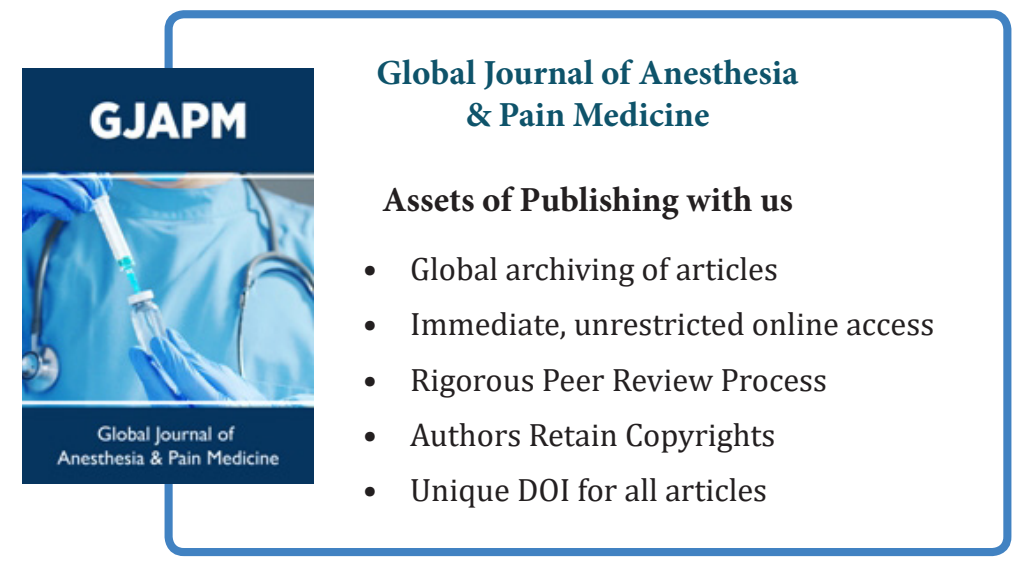

